\title{
Simulation of the active Brownian motion of a microswimmer
}

Giorgio Volpe, Sylvain Gigan, and Giovanni Volpe

Citation: American Journal of Physics 82, 659 (2014); doi: 10.1119/1.4870398

View online: http://dx.doi.org/10.1119/1.4870398

View Table of Contents: http://aapt.scitation.org/toc/ajp/82/7

Published by the American Association of Physics Teachers

\section{Articles you may be interested in}

Simulation of a Brownian particle in an optical trap

American Journal of Physics 81, 224 (2013); 10.1119/1.4772632

Life at low Reynolds number

American Journal of Physics 45, 3 (1998); 10.1119/1.10903

Dynamical density functional theory for microswimmers

The Journal of Chemical Physics 144, 024115 (2016); 10.1063/1.4939630

Internal dynamics of semiflexible polymers with active noise

The Journal of Chemical Physics 146, 154903 (2017); 10.1063/1.4981012

Brownian dynamics with hydrodynamic interactions

The Journal of Chemical Physics 69, 1352 (2008); 10.1063/1.436761

Brownian dynamics simulations with hard-body interactions: Spherical particles

The Journal of Chemical Physics 137, 164108 (2012); 10.1063/1.4761827

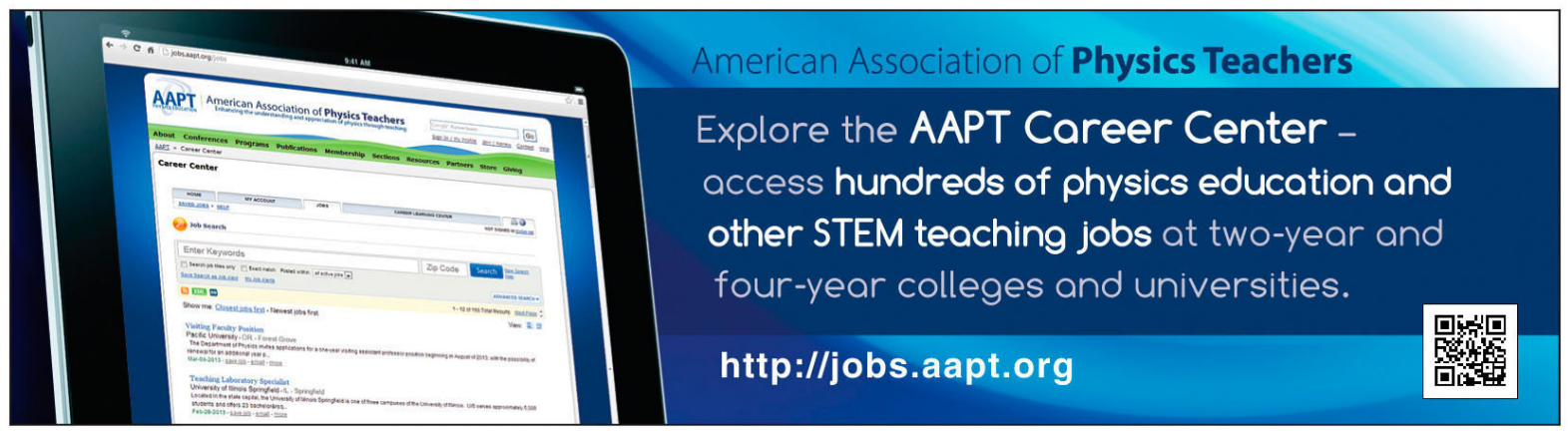




\title{
Simulation of the active Brownian motion of a microswimmer
}

\author{
Giorgio Volpe and Sylvain Gigan \\ Institut Langevin, ESPCI ParisTech, CNRS UMR7587, 1 rue Jussieu, 75005 Paris, France \\ Giovanni Volpe ${ }^{\text {a) }}$ \\ Physics Department, Bilkent University, Cankaya, 06800 Ankara, Turkey
}

(Received 19 December 2013; accepted 24 March 2014)

\begin{abstract}
Unlike passive Brownian particles, active Brownian particles, also known as microswimmers, propel themselves with directed motion and thus drive themselves out of equilibrium. Understanding their motion can provide insight into out-of-equilibrium phenomena associated with biological examples such as bacteria, as well as with artificial microswimmers. We discuss how to mathematically model their motion using a set of stochastic differential equations and how to numerically simulate it using the corresponding set of finite difference equations both in homogenous and complex environments. In particular, we show how active Brownian particles do not follow the Maxwell-Boltzmann distribution-a clear signature of their out-of-equilibrium nature-and how, unlike passive Brownian particles, microswimmers can be funneled, trapped, and sorted. $\odot 2014$ American Association of Physics Teachers.

[http://dx.doi.org/10.1119/1.4870398]
\end{abstract}

\section{INTRODUCTION}

In recent years, active Brownian motion has attracted a lot of interest from the biology and physics communities. ${ }^{1,2}$ While the motion of passive Brownian particles is driven by equilibrium thermal fluctuations, active Brownian particles, often referred to as microswimmers, are able to propel themselves, exhibiting an interplay between random fluctuations and active swimming that drives them into an out-of-equilibrium status. ${ }^{3,4}$ Several types of microscopic biological entities perform active Brownian motion; a paradigmatic example is the swimming behavior of bacteria such as Escherichia coli. ${ }^{5}$ In addition, artificial active particles hold tremendous potential as autonomous agents to localize, pick up, and deliver nanoscopic objects, e.g., in bioremediation, drug delivery, and gene therapy. ${ }^{6-8}$ Such artificial active Brownian particles propel themselves by several mechanisms, such as by a periodic deformation of their shape or by phoresis in, e.g., an electric field or a chemotactic or temperature gradient. ${ }^{9-19}$

Studying and comparing passive and active Brownian motion can provide insight into out-of-equilibrium phenomena. On the one hand, passive Brownian particles are often used to study random phenomena because their thermallydriven motion is due to random collisions with the surrounding fluid molecules; this provides a well-defined noisy background dependent on the temperature and the fluid viscosity. ${ }^{20}$ On the other hand, the motion of active particles takes them out of equilibrium. ${ }^{21}$ In order to start acquiring some first-hand experience with these phenomena, a good didactical approach is to perform numerical experiments, which have the advantage of being inexpensive and within the reach of every student with access to a computational device. Numerical experiments can also be used to introduce and complement real experiments.

In this article, we explain step by step how to model the motion of an active Brownian particle in homogenous and complex environments. First, we introduce the basic mathematical model of the motion of an active Brownian particle in a two-dimensional homogeneous environment in terms of stochastic differential equations, and solve the equations numerically using a simple finite-difference algorithm. Then, we illustrate how to simulate the motion of an active particle in a complex environment where several obstacles are present using reflective boundary conditions. Unlike passive Brownian particles, we observe that microswimmers can be funneled, trapped, and sorted by using the out-of-equilibrium nature of their motion. We provide the MATLAB programs used for these simulations as an online supplement; ${ }^{22}$ these programs can be straightforwardly adapted to the freeware SciLab $^{23}$ or GNU Octave. ${ }^{24}$

\section{MATHEMATICAL MODEL}

In a two-dimensional homogeneous environment, the motion of an active particle can be modeled as the combined action of three different processes: ${ }^{25,26}$ a random diffusion process, an internal self-propelling force, and, in the case of chiral active particles, a torque. In particular, the position $[x(t), y(t)]$ of a spherical microscopic particle with radius $R$ undergoes Brownian diffusion with translational diffusion coefficient

$$
D_{\mathrm{T}}=\frac{k_{\mathrm{B}} T}{6 \pi \eta R},
$$

where $k_{B}$ is the Boltzmann constant, $T$ the temperature, and $\eta$ the fluid viscosity. The particle self-propulsion results in a directed component of the motion with speed $v$ we will assume to be constant and with a direction that depends on the particle orientation $\varphi(t)$, as illustrated in Fig. 1(a). Finally, $\varphi(t)$ undergoes rotational diffusion with rotational diffusion coefficient

$$
D_{\mathrm{R}}=\frac{k_{\mathrm{B}} T}{8 \pi \eta R^{3}} .
$$

For a chiral active particle, $\varphi(t)$ also rotates with angular velocity $\Omega$ as a consequence of a torque acting on the particle ${ }^{27}$ in the presence of a propulsion speed $v>0$, this reorientation of the particle translates into a rotation around an effective external axis, as shown in Fig. 1(b). The sign of $\Omega$ determines the chirality of the particles. In the most general case, the resulting set of Langevin equations ${ }^{28}$ describing this motion in two dimensions is 


$$
\begin{aligned}
& \frac{d}{d t} \varphi(t)=\Omega+\sqrt{2 D_{\mathrm{R}}} W_{\varphi}, \\
& \frac{d}{d t} x(t)=v \cos \varphi(t)+\sqrt{2 D_{\mathrm{T}}} W_{x}, \\
& \frac{d}{d t} y(t)=v \sin \varphi(t)+\sqrt{2 D_{\mathrm{T}}} W_{y},
\end{aligned}
$$

where $W_{\varphi}, W_{x}$, and $W_{y}$ represent independent white noise processes. Inertial effects are neglected because typically microscopic active particles move in a low Reynolds number regime. ${ }^{29}$ In the following, we will always consider a particle with radius $R=1 \mu \mathrm{m}$ at temperature $T=300 \mathrm{~K}$ immersed in a liquid with viscosity $\eta=0.001 \mathrm{~N} \mathrm{~s} / \mathrm{m}^{2}$ (such as water); the corresponding translational diffusion coefficient is $D_{\mathrm{T}} \approx 0.22 \mu \mathrm{m}^{2} / \mathrm{s}$ and the corresponding rotational diffusion coefficient is $D_{\mathrm{R}} \approx 0.16 \mathrm{rad}^{2} / \mathrm{s}$.

\section{FINITE DIFFERENCE EQUATIONS}

The continuous-time solution $[\varphi(t), x(t), y(t)]$ to the set of stochastic differential equations given by Eqs. (3)-(5) can be approximated by a discrete-time sequence $\left[\varphi_{i}, x_{i}, y_{i}\right]$ $\approx\left[\varphi\left(t_{i}\right), x\left(t_{i}\right), y\left(t_{i}\right)\right]$ that is the solution of the corresponding set of finite difference equations evaluated at regular time steps $t_{i}=i \Delta t$, where $\Delta t$ is a sufficiently small time step. In order to derive the set of finite difference equations, the white noise factors in Eqs. (3)-(5) must be dealt with carefully, for example, by following the procedure described in Ref. 30. Explicitly, the set of finite difference equations can
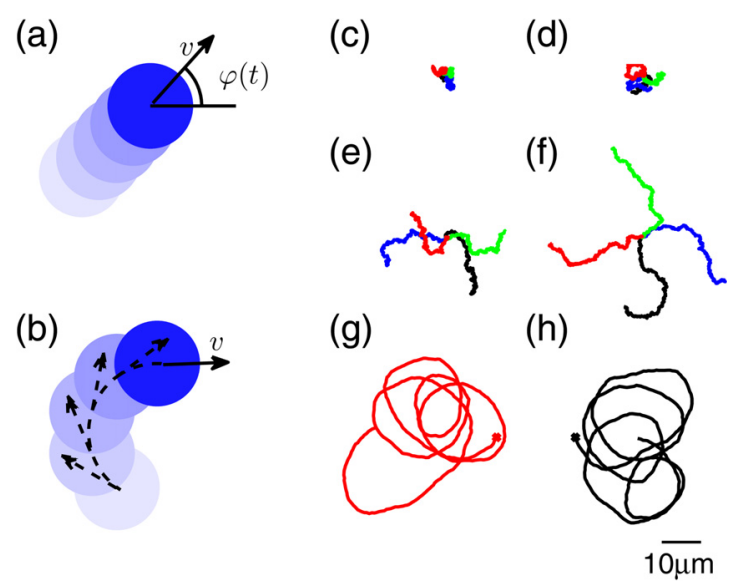

Fig. 1. (Color online) Active Brownian particles in two dimensions. (a) An active Brownian particle placed at $[x(t), y(t)]$ is characterized by an orientation $\varphi(t)$ along which it propels itself with speed $v$ while it undergoes Brownian motion in both its position and orientation. (b) A chiral active Brownian particle also has a deterministic angular velocity $\Omega$ that, if the particle's speed $v>0$, translates into a rotation around an effective external axis. Trajectories of active particles are shown at right for (c) $v=0$, (d) $v=1 \mu \mathrm{m} / \mathrm{s}$, (e) $v=2 \mu \mathrm{m} / \mathrm{s}$, and (f) $v=3 \mu \mathrm{m} / \mathrm{s}$, all for $\Omega=0$. With increasing $v$, the particles move longer distances before the direction of their motion is randomized; four different 10-s trajectories are shown for each velocity. Trajectories for chiral active particles are shown in (g) and (h), with $v=31 \mu \mathrm{m} / \mathrm{s}$ and $\Omega= \pm 3.14 \mathrm{rad} / \mathrm{s}$, starting at the position indicated by the cross and lasting $10 \mathrm{~s}$; the sign of $\Omega$ causes the trajectory to bend either (g) clockwise or (h) counterclockwise. The particles have radius $R=1 \mu \mathrm{m}$, are at temperature $T=300 \mathrm{~K}$, and are immersed in a liquid with viscosity $\eta=0.001 \mathrm{~N} \mathrm{~s} / \mathrm{m}^{2}$; the corresponding translational diffusion coefficient is $D_{\mathrm{T}} \approx 0.22 \mu \mathrm{m}^{2} / \mathrm{s}$ and the rotational diffusion coefficient is $D_{\mathrm{R}} \approx 0.16 \mathrm{rad}^{2} / \mathrm{s}$. be obtained from Eqs. (3)-(5) by carrying out the substitutions

$$
\begin{aligned}
& \varphi(t) \rightarrow \varphi_{i}, \quad x(t) \rightarrow x_{i}, \quad y(t) \rightarrow y_{i} ; \\
& \frac{d}{d t} \varphi(t) \rightarrow \frac{\varphi_{i}-\varphi_{i-1}}{\Delta t}, \quad \frac{d}{d t} x(t) \rightarrow \frac{x_{i}-x_{i-1}}{\Delta t}, \\
& \quad \frac{d}{d t} y(t) \rightarrow \frac{y_{i}-y_{i-1}}{\Delta t} ; \\
& W_{\varphi} \rightarrow \frac{w_{\varphi, i}}{\sqrt{\Delta t}}, \quad W_{x} \rightarrow \frac{w_{x, i}}{\sqrt{\Delta t}}, \quad W_{y} \rightarrow \frac{w_{y, i}}{\sqrt{\Delta t}} .
\end{aligned}
$$

Here $w_{\varphi, i}, w_{x, i}$, and $w_{y, i}$ are uncorrelated sequences of random numbers taken from a Gaussian distribution with zero mean and standard deviation 1. Many programming languages have built-in functions that directly generate such random sequences. Alternatively, it is possible to generate Gaussian random numbers from uniform random numbers between 0 and 1 using various techniques such as the BoxMüller algorithm or the Marsaglia polar algorithm. ${ }^{31}$ The numerical solution is then obtained by solving the resulting finite difference equation recursively for $\left[\varphi_{i}, x_{i}, y_{i}\right]$ using the values $\left[\varphi_{i-1}, x_{i-1}, y_{i-1}\right]$ obtained from the previous iteration:

$$
\begin{aligned}
& \varphi_{i}=\varphi_{i-1}+\Omega \Delta t+\sqrt{2 D_{\mathrm{R}} \Delta t} w_{\varphi, i}, \\
& x_{i}=x_{i-1}+v \cos \varphi_{i-1} \Delta t+\sqrt{2 D_{\mathrm{T}} \Delta t} w_{x, i}, \\
& y_{i}=y_{i-1}+v \sin \varphi_{i-1} \Delta t+\sqrt{2 D_{\mathrm{T}} \Delta t} w_{y, i} .
\end{aligned}
$$

We note that this is a first-order integration method that generalizes the Euler method to stochastic differential equations; higher-order algorithms can also be employed to obtain faster convergence of the solution. ${ }^{31}$

\section{HOMOGENEOUS ENVIRONMENT}

We start by considering non-chiral $(\Omega=0)$ active Brownian particles. When the self-propulsion speed $v$ is zero, the particle's motion is purely diffusive with diffusion constant $D_{\mathrm{T}}$; some examples of the corresponding trajectories are illustrated in Fig. 1(c). As $v$ increases we obtain active trajectories that are characterized by directed motion on short time scales, as shown in Figs. 1(d)-1(f). However, while on short time scales, the motion is dominated by their directed self-propulsion, over long time scales the orientation of the particle is randomized by the rotational diffusion. The time scale of the rotational diffusion is given by $\tau_{\mathrm{R}}=1 / D_{\mathrm{R}}$ $\approx 6.25 \mathrm{~s}$ for the particles we consider in this article. These qualitative considerations can be made more precise by calculating the mean square displacement $\operatorname{MSD}(\tau)$ of the particle's motion. The $\operatorname{MSD}(\tau)$ quantifies how a particle moves from its initial position, and can be calculated from a trajectory as

$$
\operatorname{MSD}(\tau)=\left\langle[x(t+\tau)-x(t)]^{2}+[y(t+\tau)-y(t)]^{2}\right\rangle .
$$

Numerically, the MSD can be calculated from a trajectory $\left[x_{n}, y_{n}\right]$ sampled at discrete times $t_{n}$ with a time step $\Delta t$ as $^{30}$

$$
\operatorname{MSD}(m \Delta t)=\left\langle\left(x_{n+m}-x_{n}\right)^{2}+\left(y_{n+m}-y_{n}\right)^{2}\right\rangle .
$$


For ballistic motion $\operatorname{MSD}(\tau)$ is proportional to $\tau^{2}$ while for diffusive motion it is proportional to $\tau$. As can be seen in Fig. 2(a), when $v \neq 0$ the $\operatorname{MSD}(\tau)$ deviates from a diffusive behavior on short time scales and exhibits an enhanced effective diffusion over long time scales.

The theoretical value of $\operatorname{MSD}(\tau)$ is given by the formula $^{12,32}$

$$
\operatorname{MSD}(\tau)=\left[4 D_{\mathrm{T}}+v^{2} \tau_{\mathrm{R}}\right] \tau+\frac{v^{2} \tau_{\mathrm{R}}^{2}}{2}\left[e^{-2 \tau / \tau_{\mathrm{R}}}-1\right],
$$

which is essentially the Ornstein-Uhlenbeck formula for the MSD of a Brownian particle with inertia, ${ }^{33}$ describing the transition from the ballistic regime to the diffusive regime although at a much shorter time scale than for active particles. ${ }^{30}$ From Eq. (14), we find that for $\tau \gg \tau_{\mathrm{R}}$ the effective particle diffusion is $D_{\text {eff }}=D_{\mathrm{T}}+v^{2} \tau_{\mathrm{R}} / 4$, and for $\tau \ll \tau_{\mathrm{R}}$ the particle motion is ballistic with $\operatorname{MSD}(\tau) \propto v^{2} \tau^{2}$. The theoretical $\operatorname{MSD}(\tau)$ calculated using Eq. (14) is shown as the solid lines in Fig. 2(b). While the features of the MSD of a microswimmer are well captured by Eq. (14), there can be deviations due to the microscopic dynamics of the microswimmer. ${ }^{12}$

We now consider the case of chiral active Brownian particles. There are many natural examples of chiral microswimmers. For example, E. coli bacteria and spermatozoa undergo helicoidal motion, which becomes two-dimensional chiral active Brownian motion when moving near boundaries. ${ }^{34-37}$ Figure $1(\mathrm{~g})$ shows the simulated trajectory of a chiral particle with $\Omega=+3.14 \mathrm{rad} / \mathrm{s}$ and $v=31 \mu \mathrm{m} / \mathrm{s}$; it bends clockwise, tracking almost circular trajectories that are modified by Brownian fluctuations. Changing the chirality sign $(\Omega=-3.14 \mathrm{rad} / \mathrm{s})$ results in similar trajectories that bend counterclockwise [Fig. 1(h)].
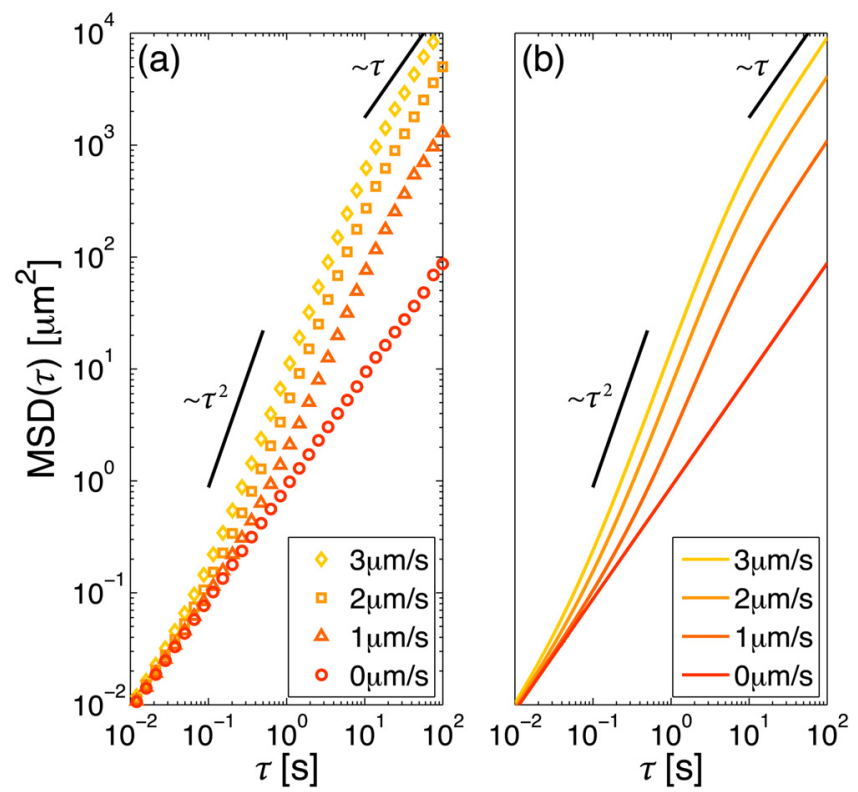

Fig. 2. (Color online) (a) Numerically calculated and (b) theoretical mean square displacement for active Brownian particles with self-propulsion velocity $v=0$ (circles), $v=1 \mu \mathrm{m} / \mathrm{s}$ (triangles), $v=2 \mu \mathrm{m} / \mathrm{s}$ (squares), and $v=3 \mu \mathrm{m} / \mathrm{s}$ (diamonds); in all cases, $\Omega=0$. For passive Brownian particles $(v=0)$ the motion is always diffusive $(\operatorname{MSD}(\tau) \propto \tau)$, while for active Brownian particles the motion is ballistic on short time scales $\left(\operatorname{MSD}(\tau) \propto \tau^{2}\right.$ for $\left.\tau \ll \tau_{R}\right)$ and then becomes diffusive on long time scales $\left(\operatorname{MSD}(\tau) \propto \tau\right.$ for $\tau \gg \tau_{R}$ ) with an enhanced diffusion constant. (a)

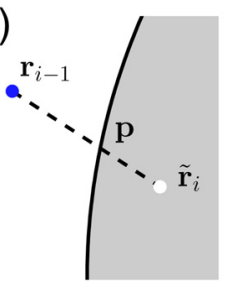

(b)

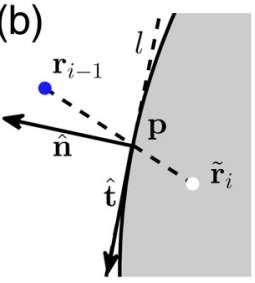

(c)

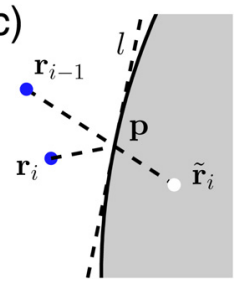

Fig. 3. Implementation of reflective boundary conditions. At each time step, the algorithm (a) checks whether a particle has moved inside an obstacle; if so: (b) the boundary of the obstacle is approximated by its tangent $l$ at the point $\mathbf{p}$ where the particle entered the obstacle, and (c) the particle position is reflected on this line.

\section{COMPLEX ENVIRONMENTS}

\section{A. Reflective boundaries}

So far we have considered particles that move only in a homogeneous environment. However, self-propelled particles often move in patterned environments, e.g., inside the intestinal tract, which provides the natural habitat of E. coli, ${ }^{5}$ or through porous polluted soils, where chemotactic bacteria spread during bioremediation. ${ }^{7}$ In a similar fashion, artificial microswimmers must also reliably perform their tasks in complex surroundings, e.g., inside lab-on-a-chip devices or in living organisms. ${ }^{38}$ When self-propelled particles move through a patterned environment, frequent encounters with obstacles will occur. Whenever an active particle contacts an obstacle, it slides along the obstacle until its orientation points away from it. Numerically, this process can be modeled using reflective boundaries, as shown in Fig. 3.

The concrete implementation of the reflective boundary condition is realized by updating at each time step the particle position from $\mathbf{r}_{i-1}=\left[x_{i-1}, y_{i-1}\right]$ to $\mathbf{r}_{i}=\left[x_{i}, y_{i}\right]$ according to the following algorithm:

1. tentatively update the particle position to $\tilde{\mathbf{r}}_{i}=\left[\tilde{x}_{i}, \tilde{y}_{i}\right]$ according to Eqs. (9)-(11);

2. if $\tilde{\mathbf{r}}_{i}$ is not inside any obstacle, set $\mathbf{r}_{i}=\tilde{\mathbf{r}}_{i}$ and move on to the next time step;

3. otherwise, if $\tilde{\mathbf{r}}_{i}$ is inside some obstacle, as depicted in Fig. 3(a):
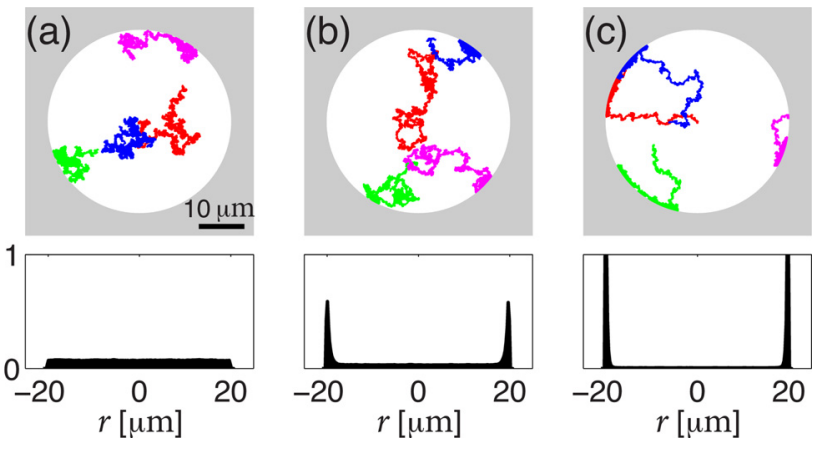

Fig. 4. (Color online) Trajectories over a 10-s time period of Brownian particles moving within a circular pore (diameter $40 \mu \mathrm{m}$ ) with reflective boundaries at self-propulsion velocity (a) $v=0$, (b) $v=5 \mu \mathrm{m} / \mathrm{s}$, and (c) $v=10 \mu \mathrm{m} / \mathrm{s}$, all for $\Omega=0$. The histograms on the bottom show the probability distribution along a diameter of the circular pore. While the probability is uniform across the whole pore for passive Brownian particles, the probability increases towards the walls for active Brownian particles as $v$ increases. 

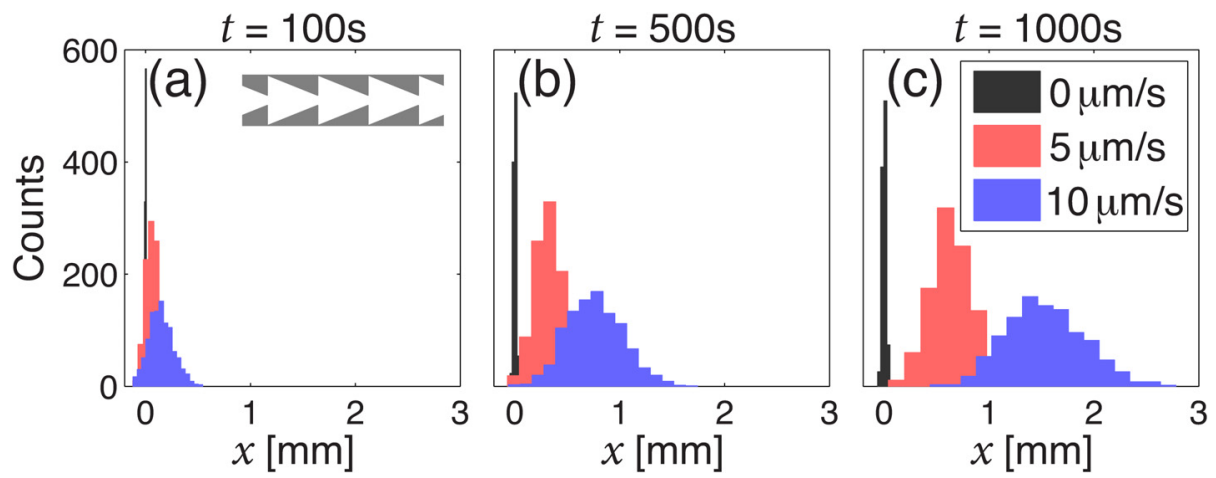

Fig. 5. (Color online) Rectification of active Brownian motion in an asymmetric ratchet-like microchannel. A segment of the channel, whose dent is 10- $\mu$ m long, is represented by the grey structure in the inset of (a); the walls of the channel are infinitely extended. The distributions of passive (black histograms) and active (gray/colored histograms) Brownian particles released at time $t=0$ from position $x=0$ are plotted at times (a) $t=100 \mathrm{~s}$, (b) $t=500 \mathrm{~s}$ and (c) $t=1000 \mathrm{~s}$. The $x$-axis gives the distance from the fixed, constant starting position of the particles. The higher the self-propulsion velocity, the farther the active particles travel along the channel. Each histogram is calculated using 1000 particle trajectories.

(a) calculate the intersection point $\mathbf{p}=\left[x_{\mathrm{p}}, y_{\mathrm{p}}\right]$ between the boundary and the line from $\mathbf{r}_{i-1}$ to $\tilde{\mathbf{r}}_{i}$;

(b) calculate the straight line $l$ tangent to the obstacle at $\mathbf{p}$ with tangent unit vector $\hat{\mathbf{t}}$ and normal unit vector $\hat{\mathbf{n}}$ (outgoing from the obstacle), as shown in Fig. 3(b);

(c) calculate $\mathbf{r}_{i}$ by reflecting $\tilde{\mathbf{r}}_{i}$ on $l$ so that

$$
\mathbf{r}_{i}=\tilde{\mathbf{r}}_{i}-2\left[\left(\tilde{\mathbf{r}}_{i}-\mathbf{p}\right) \cdot \hat{\mathbf{n}}\right] \hat{\mathbf{n}}
$$

where $\left(\tilde{\mathbf{r}}_{i}-\mathbf{p}\right) \cdot \hat{\mathbf{n}}$ is the distance between $\tilde{\mathbf{r}}_{i}$ and $l$.

The crucial prerequisite for this numerical approach to work is that the average spatial increment of a simulated trajectory is small compared to the characteristic length scale of the obstacles. This condition permits one to consider only one boundary at a time and also to approximate the boundary with its tangent straight line. If the time step $\Delta t$ is too large, this approach can lead to some numerical instability around sharp corners in the boundaries, where multiple reflections may take place, or on an obstacle wall that is too thin, where the particle's trajectory could unnaturally pass through the obstacle.

\section{B. Non-Boltzmann probability distribution in a pore}

As a first example of a complex environment, we consider a microswimmer confined within a circular pore, as shown in Fig. 4. Figure 4(a) shows four 10-s trajectories of passive
Brownian particles $(v=0)$, which are seen to explore the configuration space within the pore uniformly. By contrast, active Brownian particles, shown in Figs. 4(b) $(v=5 \mu \mathrm{m} / \mathrm{s})$ and $4(\mathrm{c})(v=10 \mu \mathrm{m} / \mathrm{s})$, tend to spend more time at the pore boundaries. When a microswimmer encounters a boundary, it keeps on pushing against the boundary and diffusing along the cavity perimeter until the rotational diffusion orients the propulsion of the particle towards the interior of the pore. The chance that the active particle encounters the pore boundary in one of its straight runs increases as its velocity increases. These observations can be made more quantitative by using the particle probability distribution. The histograms at the bottom of Fig. 4 each show a section of this probability distribution along a diameter of the pore. We see that the probability of finding the particle at the boundaries increases with the particle's self-propulsion velocity.

That active Brownian particles tend to accumulate at the boundaries of a pore is a sign of the fact that active particles are out of equilibrium. For a passive Brownian particle in thermal equilibrium with its environment, the probability of finding the particle at a given position in the pore $p(x, y)$ is connected to the Boltzmann potential $V(x, y)$ by the Maxwell-Boltzmann relation $p(x, y) \propto \exp \left[-V(x, y) /\left(k_{\mathrm{B}} T\right)\right]$. In the case presented in Fig. 4, there are no external forces acting on the particle and, therefore, $V(x, y)$ and the corresponding Maxwell-Boltzmann distribution are constant, as seen in Fig. 4(a). However, the fact that the distributions in (a)

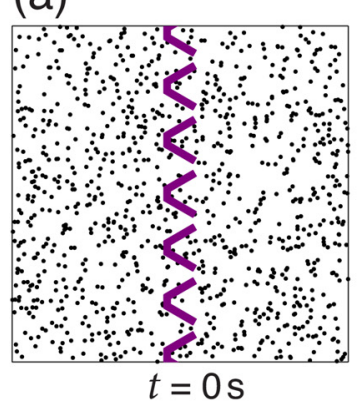

(b)

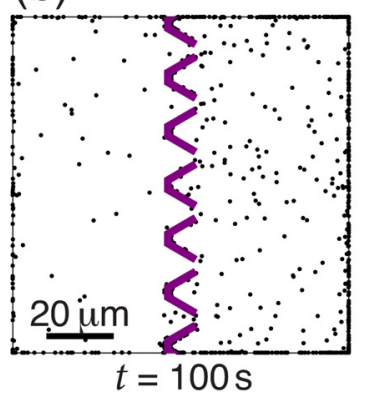

(c)

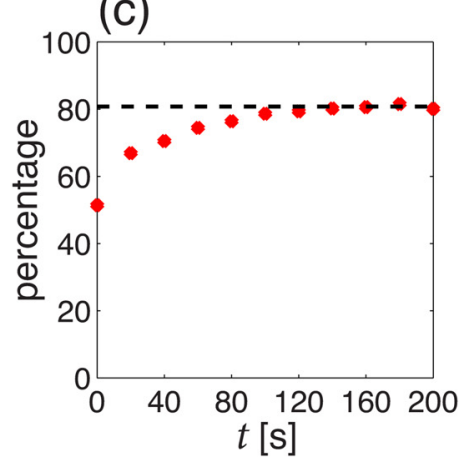

Fig. 6. Segregation of active particles $(v=10 \mu \mathrm{m} / \mathrm{s})$ using a series of wedges (dark structures), whose walls are $2 \mu \mathrm{m}$ thick. (a) At $t=0$, the active particles (black dots) are uniformly distributed across the square; (b) at $t=100 \mathrm{~s}$, most of the active particles have concentrated to the right of the wedges. (c) Percentage of the total particles on the right of the wedges (dots) as a function of time; the percentage stabilizes at around $80 \%$ (black dashed line) after the first $100 \mathrm{~s}$. 

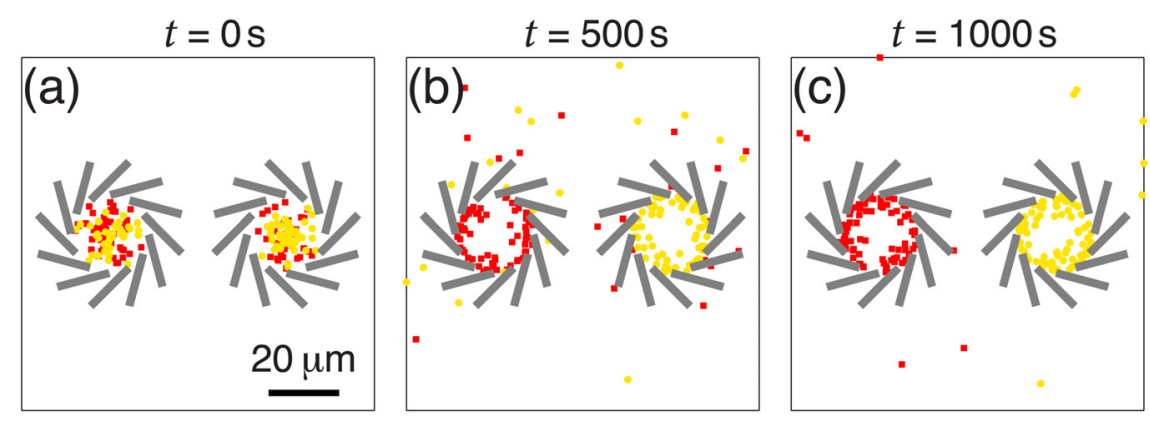

Fig. 7. (Color online) Sorting of chiral microswimmers ( $v=31 \mu \mathrm{m} / \mathrm{s}$ and $\Omega= \pm 3.14 \mathrm{rad} / \mathrm{s}$ ) with chiral "flowers" (gray rectangles, thickness $2 \mu \mathrm{m}$ ). (a) At $t=0$ a balanced mixture of active particles with opposite chiralities is released inside two chiral flowers with opposite chirality. As time progresses, shown in (b) and (c), the active particles rotating counterclockwise (darker squares) are trapped in the left chiral flower, while the particles rotating clockwise (lighter circles) are trapped in the right chiral flower.

Figs. 4(b) and 4(c) are not constant, despite the constant potentials, is a clear deviation from the Maxwell-Boltzmann distribution, thus indicating the out-of-equilibrium nature of active Brownian particles.

\section{Motion rectification in a microchannel}

The motion of active particles can be rectified by a patterned microchannel. For example, the inset of Fig. 5(a) shows an example of such a microchannel, decorated with a series of asymmetric dents on both its walls. A group of passive Brownian particles released at time $t=0$ from position $x=0$ diffuses symmetrically around the initial position (black histograms in Fig. 5). By contrast, a group of active Brownian particles is funneled by the channel in such a way that an average directed motion is imposed on the particles, as can be seen in the colored histograms in Fig. 5. The rectification is more pronounced when the self-propulsion velocity is higher. This and similar effects have been proposed to sort microswimmers on the basis of their velocity, ${ }^{26}$ to trap microswimmers in moving edges, ${ }^{39}$ and to deliver microscopic cargoes to a given location. ${ }^{19}$

\section{Trapping by asymmetric barriers}

Because active particles are not in thermal equilibrium with their environment, it is possible to use the features of the environment to perform complex tasks on active particles such as separating, trapping, or sorting them on the basis of their swimming properties. For example, Fig. 6 shows the segregation of active particles within a $100-\mu \mathrm{m}$-side square box divided into two parts by a series of wedges; this situation was first proposed in Ref. 40. At $t=0$, the active particles are homogeneously distributed in the box [Fig. 6(a)], while after $100 \mathrm{~s}$ most of the active particles concentrate in the right portion of the box [Fig. 6(b)]. The selectivity of this process depends on the system parameters, such as the size and shape of the wedges and the drift velocity of the microswimmers. ${ }^{39,40}$ Figure 6(c) shows the percentage of active particles in the right portion of the box as a function of time; with our system parameters, the distribution quickly approaches a plateau of around $80 \%$.

\section{E. Chiral particle separation}

Active particles can also be separated on the basis of their chirality. ${ }^{26,41}$ This is a particularly interesting option because it may provide a better technique to separate molecules with opposite chirality by chemically coupling them to chiral propellers, sorting the resulting chiral microswimmers, and finally detaching the propellers. Such techniques could be applied in the biochemical and pharmaceutical industry where often only one specific chirality is desired. ${ }^{42}$ Figure 7 shows a possible approach to sorting active particles based on the sign of their motion chirality in the presence of some chiral patterns in the environment, such as an arrangement of tilted rectangles along a circle forming a chiral "flower." We use two of these flowers with opposite chiralities enclosed in a $100-\mu \mathrm{m}$-side box where the particles can move freely. We start at time $t=0$ with a balanced mixture of active particles with opposite chiralities placed inside each flower [Fig. 7(a)]. As time passes, most of the microswimmers rotating counterclockwise (clockwise) escape the right (left) chiral flower, while those with the opposite chirality remain trapped. At $t=1000 \mathrm{~s}$ most of the microswimmers are stably trapped, as shown in Fig. 7(c).

\section{FURTHER NUMERICAL EXPERIMENTS}

The approach described in this article can be generalized to more complex situations. In particular, it is interesting to consider the case of an active spherical particle moving in three dimensions, where the particle position is described by three coordinates and its orientation by two angles. ${ }^{43}$ Another interesting generalization is to non-spherical active particles; for example, rods are a more accurate model for bacteria. This generalization requires the use of diffusion matrices instead of diffusion coefficients, as described in Ref. 44. Finally, it is also interesting to consider the case of multiple particles interacting with each other, for example, by Yukawa or Lennard-Jones potentials. This can be implemented using molecular dynamics algorithms. ${ }^{45}$

\section{ACKNOWLEDGMENTS}

This work was partially funded by the European Research Council (Grant No. 278025), by the Scientific and Technological Research Council of Turkey (TUBITAK) under Grant Nos. 112T235 and 113Z556, COST Actions MP1205 and IC1208, and Marie Curie Career Integration Grant (MC-CIG) under Grant No. PCIG11 GA-2012-321726.

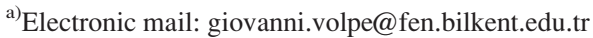

${ }^{1}$ S. J. Ebbens and J. R. Howse, "In pursuit of propulsion at the nanoscale," Soft Matter 6, 726-738 (2010).

${ }^{2}$ W. C. K. Poon, "From Clarkia to Escherichia and Janus: The physics of natural and synthetic active colloids," e-print arXiv:1306.4799 (2013).
} 
${ }^{3}$ U. Erdmann, W. Ebeling, L. Schimansky-Geier, and F. Schweitzer, "Brownian particles far from equilibrium," Eur. Phys. J. B: Condens. Matt. Comp. Sys. 15, 105-113 (2000).

${ }^{4}$ P. Hänggi and F. Marchesoni, "Artificial Brownian motors: Controlling transport on the nanoscale," Rev. Mod. Phys. 81, 387-442 (2009).

${ }^{5}$ H. C. Berg, E. coli in Motion (Springer, Heidelberg, 2004).

${ }^{6}$ D. B. Weibel, P. Garstecki, D. Ryan, W. R. DiLuzio, M. Mayer, J. E. Seto, and G. M. Whitesides, "Microoxen: Microorganisms to move microscale loads,” Proc. Natl. Acad. Sci. U.S.A. 102, 11963-11967 (2005).

${ }^{7}$ R. M. Ford and R. W. Harvey, "Role of chemotaxis in the transport of bacteria through saturated porous media," Adv. Water Res. 30, 1608-1617 (2007).

${ }^{8}$ W. Yang, V. R. Misko, K. Nelissen, M. Kong, and F. M. Peeters, "Using self-driven microswimmers for particle separation," Soft Matter 8, 5175-5179 (2012).

${ }^{9}$ R. Golestanian, T. B. Liverpool, and A. Ajdari, "Propulsion of a molecular machine by asymmetric distribution of reaction products," Phys. Rev. Lett. 94, 220801-1-4 (2005).

${ }^{10}$ W. F. Paxton, A. Sen, and T. E. Mallouk, "Motility of catalytic nanoparticles through self-generated forces," Chem. Eur. J. 11, 6462-6470 (2005).

${ }^{11}$ R. Dreyfus, J. Baudry, M. L. Roper, M. Fermigier, H. A. Stone, and J. Bibette, "Microscopic artificial swimmers," Nature 437, 862-865 (2005).

${ }^{12}$ J. R. Howse, R. A. L. Jones, A. J. Ryan, T. Gough, R. Vafabakhsh, and R. Golestanian, "Self-motile colloidal particles: From directed propulsion to random walk,” Phys. Rev. Lett. 99, 048102-1-4 (2007).

${ }^{13}$ P. Tierno, R. Golestanian, I. Pagonabarraga, and F. Sagués, "Magnetically actuated colloidal microswimmers," J. Phys. Chem. B 112, 16525-16528 (2008).

${ }^{14}$ J. Palacci, C. Cottin-Bizonne, C. Ybert, and L. Bocquet, "Sedimentation and effective temperature of active colloidal suspensions," Phys. Rev. Lett. 105, 088304-1-4 (2010).

${ }^{15}$ M. N. Popescu, S. Dietrich, M. Tasinkevych, and J. Ralston, "Phoretic motion of spheroidal particles due to self-generated solute gradients," Eur. Phys. J. E 31, 351-367 (2010).

${ }^{16}$ G. Volpe, I. Buttinoni, D. Vogt, H.-J. Kümmerer, and C. Bechinger, "Microswimmers in patterned environments," Soft Matter 7, 8810-8815 (2011).

${ }^{17}$ A. Búzás, L. Kelemen, A. Mathesz, L. Oroszi, G. Vizsnyiczai, T. Vicsek, and P. Ormos, "Light sailboats: Laser driven autonomous microrobots," Appl. Phys. Lett. 101, 041111-1-3 (2012).

${ }^{18}$ I. Buttinoni, G. Volpe, F. Kümmel, G. Volpe, and C. Bechinger, "Active Brownian motion tunable by light," J. Phys.: Condens. Matter 24, 2841291-6 (2012).

${ }^{19}$ N. Koumakis, A. Lepore, C. Maggi, and R. Di Leonardo, "Targeted delivery of colloids by swimming bacteria," Nat. Commun. 4, 2588-1-6 (2013).

${ }^{20}$ D. Babič, C. Schmitt, and C. Bechinger, "Colloids as model systems for problems in statistical physics," Chaos 15, 026114-1-6 (2005).

${ }^{21}$ F. Schweitzer, Brownian Agents and Active Particles: Collective Dynamics in the Natural and Social Sciences (Springer, Heidelberg, 2007).

${ }^{22}$ See supplementary material at http://dx.doi.org/10.1119/1.4870398 for the Matlab codes.

${ }^{23}$ Scilab, open source software for numerical computation, $<$ www.scilab.org/>.
${ }^{24}$ GNU Octave software, <www.gnu.org/software/octave/ $>$.

${ }^{25} \mathrm{~S}$. van Teeffelen and H. Löwen, "Dynamics of a Brownian circle swimmer," Phys. Rev. E 78, 020101-1-4 (2008).

${ }^{26}$ M. Mijalkov and G. Volpe, "Sorting of chiral microswimmers," Soft Matter 9, 6376-6381 (2013).

${ }^{27}$ F. Kümmel, B. Ten Hagen, R. Wittkowski, I. Buttinoni, R. Eichhorn, G. Volpe, H. Löwen, and C. Bechinger, "Circular motion of asymmetric selfpropelling particles," Phys. Rev. Lett. 110, 198302-1-5 (2013).

${ }^{28}$ W. T. Coffey, Yu. P. Kalmykov, and J. T. Waldron, The Langevin Equation: With Applications to Stochastic Problems in Physics, Chemistry, and Electrical Engineering (World Scientific, New York, 2004).

${ }^{29}$ E. M. Purcell, "Life at low Reynolds number," Am. J. Phys. 45, 3-11 (1977).

${ }^{30} \mathrm{G}$. Volpe and G. Volpe, "Simulation of a Brownian particle in an optical trap," Am. J. Phys. 81, 224-230 (2013).

${ }^{31}$ P. E. Kloeden and R. A. Pearson, Numerical Solution of Stochastic Differential Equations (Springer, Heidelberg, 1999).

${ }^{32} \mathrm{~K}$. Franke and H. Gruler, "Galvanotaxis of human granulocytes: Electric field jump studies,” Eur. Biophys. J. 18, 334-346 (1990).

${ }^{33}$ G. E. Uhlenbeck and L. S. Ornstein, "On the theory of the Brownian motion," Phys. Rev. 36, 823-841 (1930).

${ }^{34}$ E. Lauga, W. R. DiLuzio, G. M. Whitesides, and H. A. Stone, "Swimming in circles: Motion of bacteria near solid boundaries," Biophys. J. 90, 400-412 (2006)

${ }^{35}$ B. M. Friedrich and F. Jülicher, "Steering chiral swimmers along noisy helical paths," Phys. Rev. Lett. 103, 068102-1-4 (2009).

${ }^{36}$ R. Di Leonardo, D. Dell'Arciprete, L. Angelani, and V. Iebba, "Swimming with an image," Phys. Rev. Lett. 106, 038101-1-4 (2011).

${ }^{37}$ Ting -Wei Su, Liang Xue, and Aydogan Ozcan, "High-throughput lensfree 3D tracking of human sperms reveals rare statistics of helical trajectories," Proc. Natl. Acad. Sci. U.S.A. 109, 16018-16022 (2012).

${ }^{38}$ C. D. Chin, V. Linder, and S. K. Sia, "Lab-on-a-chip devices for global health: Past studies and future opportunities," Lab Chip 7, 41-57 (2007).

${ }^{39}$ A. Kaiser, K. Popowa, H. H. Wensink, and H. Löwen, "Capturing selfpropelled particles in a moving microwedge,” Phys. Rev. E 88, 0223111-9 (2013).

${ }^{40}$ M. B. Wan, C. J. O. Reichhardt, Z. Nussinov, and C. Reichhardt, "Rectification of swimming bacteria and self-driven particle systems by arrays of asymmetric barriers," Phys. Rev. Lett. 101, 018102-1-4 (2008).

${ }^{41}$ C. Reichhardt and C. J. O. Reichhardt, "Dynamics and separation of circularly moving particles in asymmetrically patterned arrays," Phys. Rev. E 88, 042306-1-10 (2013).

${ }^{42}$ S. Ahuja, editor, Chiral Separation Methods for Pharmaceutical and Biotechnological Products (John Wiley and Sons, Inc., Hoboken, NJ, 2011).

${ }^{43}$ T. Carlsson, T. Ekholm, and C. Elvingson, "Algorithm for generating a Brownian motion on a sphere," J. Phys. A: Math. Theor. 43, 505001-1-10 (2010).

${ }^{44}$ M. X. Fernandes and J. García de la Torre, "Brownian dynamics simulation of rigid particles of arbitrary shape in external fields," Biophys. J. 83, 3039-3048 (2002).

${ }^{45}$ D. Frenkel and B. Smit, Understanding Molecular Simulation: From Algorithms to Applications (Elsevier, Amsterdam, 2001). 